\title{
Association of ulcerative colitis and Clostridium difficile infection at pediatric age - case presentation
}

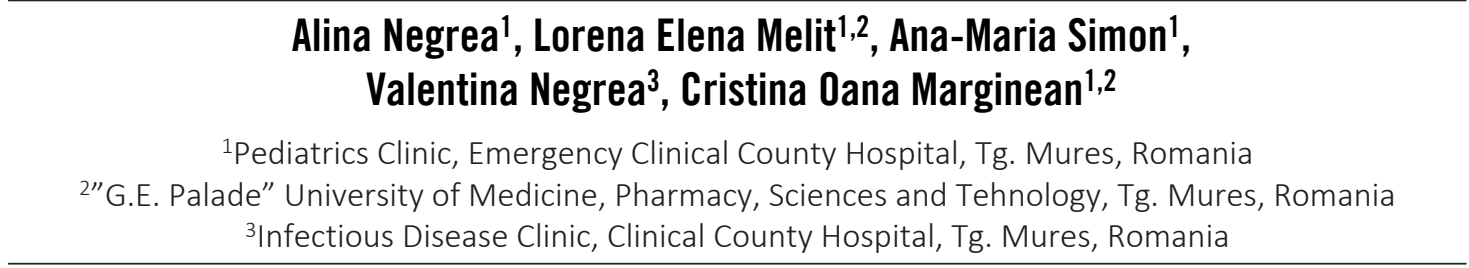

\begin{abstract}
Introduction. Ulcerative colitis (UC) is a more severe pathology in children than adults, with an increasing incidence in young ages. The most common exacerbations are due to Clostridium difficile (CD) infection and can lead to severe complications.

Case presentation. We present the case of a 6-year-old patient diagnosed with ulcerative colitis at the age of 5, admitted in our Clinic for watery diarrheic stools with blood, abdominal pain, anorexia, and weight loss. Laboratory tests at the time of admission revealed anemia, leukocytosis with neutrophilia, hydro-electrolytic disorders, and severe hypoalbuminemia. The A and B toxin detection test for CD in feces was positive, stool culture was negative. Anamnestic, clinical and paraclinical data established the diagnosis of CD infection associated with exacerbation of UC. Initially, the evolution under pulse therapy with Solu-Medrol and Metronidazole by vein was slightly favorable, but with a period of decline 5 days after the per oral administration of corticosteroids. Therefore, it was necessary to reinitiate intravenous corticosteroids associating also Vancomycin orally. Under the established treatment the symptomatology improved, with the normalization of the laboratory parameters and after 21 days of admission the patient was discharged.

Conclusions. The coexistence of CD infection in pediatric patients with UC remains a real challenge, due to the high rate of recurrence and higher mortality compared to the general population. The undulating evolution under treatment, as well as the psychological profile of the patient, have customized the case.
\end{abstract}

Keywords: ulcerative colitis, exacerbation, Clostridium difficile, management
Abbreviations
IBD - inflammatory bowel diseases
$\mathrm{UC}$ - ulcerative colitis
$\mathrm{CD}$ - Clostridium difficile
CDI - Clostridium difficile infection

\author{
$\mathrm{CRP}$ - C-reactive protein \\ $\mathrm{Hg}$ - hemoglobin \\ Htc - hematocrit
}
ESR - erythrocyte sedimentation rate

\section{INTRODUCTION}

Ulcerative colitis (UC) and Crohn's disease belong to a heterogeneous group of chronic, idiopathic, nonspecific inflammatory disorders of the gastrointestinal tract, which are known as inflammatory bowel diseases (IBD). The term unclassified inflammatory bowel disease is used if $\mathrm{UC}$ and Crohn disease cannot be differentiated according to the ESPGHAN criteria (1). The etiology and pathogenesis of this diseases are multifactorial, involving environmental, immunological, genetic and intestinal microbiota factors (2). The interaction between these factors is extremely important in the onset of the disease. Although stress and anxiety may favor UC exacerbation, their involvement in the development 
of the disease is not clear, but definitely contribute to a rather important extent to the evolution of this pathology (3). UC affects only the colon and rectum. Depending on the extent of the lesions, the topographic diagnosis, according to the Paris classification, consists of: rectitis - E1 is affected only the rectum, left colitis - E2 distal of the splenic flexure, extended colitis - E3 to or upstream of the hepatic flexura - E4 (4). Inflammation is continuous and limited to the mucosa. However, extensive colitis is the most common form of ulcerative colitis in children, as opposed to adults $(1,4)$. A quarter of cases of inflammatory bowel disease are diagnosed in children under the age of 18 , the average age of onset being between 10 and 12 years with a tendency to decrease in recent years (5). The incidence of pediatric onset UC is increasing, being very different across the globe. The industrialized countries of Western Europe, Scandinavia, North America are at the top of the list, with one case in 250 healthy people (6). The prevalence of UC ranges between 0.2 and 6.7 for 1,000 children $(5,6)$. UC is characterized predominantly by digestive manifestations, such as abdominal pain, diarrheal stools with or without pathological elements, tenesmus, rectal bleeding, nausea, vomiting, weight loss (7); however, extraintestinal manifestations may also be present, such as: arthritis, skin lesions, liver damage or eye damage (8). The therapeutic approach of pediatric patients with UC is complex and must be individualized according to the patient's particularities, the form of the disease but also the presence or absence of possible complications. The goals of therapy consist in obtaining and maintaining remission, avoiding complications, but also ensuring a quality of life corresponding to the patient's age (1). The evolution of UC consists of a succession of flares and remissions. According to the latest data from the literature, $20 \%$ of exacerbations are due to $\mathrm{CD}$ infection with significant increased morbidity (9).

$\mathrm{CD}$ is a Gram positive, anaerobic bacillus, whose virulence is due to the release of toxins $\mathrm{A}$ and $\mathrm{B}$ into the intestine. It is known that several categories of patients are at increased risk of infection including patients treated with broad-spectrum antibiotics over a long period of time, long time hospitalized, immunocompromised patients, or those receiving treatment with proton pump inhibitors or $\mathrm{H} 2$ receptor antagonists (10). The incidence of $\mathrm{CD}$ in pediatric patients with IBD is not clear, suggesting that $24.7 \%$ of UC cases in children may be associated with this infection compared to $9 \%$ in children without IBD (11). Common symptoms of mild and moderate forms consist of watery diarrhea, fever and mild abdominal pain (12). Metronidazole is the first line of treatment for the first episode of infection with mild forms, while Vancomycin orally is recommended for moderate and severe ones. The association between Vancomycin orally Metronidazole by vein is recommended in severe cases or with systemic complications $(13,14)$.

The purpose of this presentation is to highlight the challenges arising in the diagnosis and management of a patient with UC and its exacerbation in the context of an infection with $\mathrm{CD}$.

\section{CASE REPORT}

\section{Presenting concerns}

We present the case of a 6-year-old male diagnosed 7 months ago with UC, for which he required multiple admissions, but without adequate treatment at home due to the parent's lack of compliance. The current symptomatology began about two weeks before admission with abdominal pain, watery diarrheic stools with blood, fever, anorexia and weight loss.

\section{Clinical findings}

Upon admission, the clinical exam revealed hyposthenia with influenced general status, ailing facies, pallor, diminished skin turgor, edema in the bilateral lower limbs, with the sign of positive well, white deposits on the tongue, balanced cardio-respiratory, no abdominal contracture, abdominal tenderness at superficial and deep palpation, accelerated intestinal transit.

\section{Diagnostic focus and assessment}

Initial investigations revealed anemia $(\mathrm{Hg}: 9.5 \mathrm{~g} / \mathrm{dl}$; Htc: $27.9 \%)$, leukocytosis $\left(24,670 / \mathrm{mm}^{3}\right)$, neutrophilia (73.9\%), increased C-reactive protein (CRP: 148.62 $\mathrm{mg} / \mathrm{l}$ ), hyponatremia and hypopotassemia (sodium: $128 \mathrm{mmol} / 1$, potassium: $2.81 \mathrm{mmol} / \mathrm{l})$, hypoalbuminemia (albumin: $1.7 \mathrm{~g} / \mathrm{dl}$ ), hypoproteinemia (4.35 $\mathrm{g} / \mathrm{dl}$ ). Taking into account the clinical picture, we collected stool samples for the detection of CD A and B toxins, with a positive result for both exotoxins, the stool culture being negative. Thus, we established the 
diagnosis of CDI associated with exacerbation of UC.

\section{Therapeutic focus and assessment}

In view of the trenchant evolution, pulsed therapy with Solu-Medrol, Metronidazole iv for 7 days, due to low digestive tolerance, subsequently orally up to 14 days, symptomatic (Smecta, Hydrasec, Enterol), and human albumin were administered. The patient's clinical and paraclinical evolution during intravenous corticosteroids and antibiotic was slightly favorable with improvement in symptomatology. On the $5^{\text {th }}$ day after switching to oral corticosteroids, diarrheal stools with blood reappeared, with an increase in inflammatory markers, leukocytosis with neutrophilia. Therefore, we reintroduced corticosteroids by vein, associated Vancomycin orally and immunomodulator treatment with Azathioprine. Further, the evolution was slowly favorable, and the patient was discharged after 21 days with oral corticosteroids. Taking into account the fact that the patient had anxiety disorders, irritability, episodes of functional abdominal pain, somatization, being reluctant to the diet, we requested a pediatric neuropsychiatry consult, establishing the diagnosis of psycho-organic syndrome with anxious elements and separation anxiety.

\section{Follow-up and outcome}

The parent's poor compliance and their stable residence in another country led to the impossibility of following the evolution of this case due to the failure to attend the recommended periodic checks. Moreover, reduced compliance could be a great burden in terms of the patient's prognosis.

\section{DISCUSSIONS}

Recent epidemiological studies suggest an increase in the incidence of IBD in young ages $(15,16)$. Depending on the age at onset, IBD can be classified as pediatric onset in children under 17 years of age, early onset under 10 years of age, very early under 6 years of age, and neonatal onset $(17,18)$. Studies suggested that neonatal or infantile onset have a more severe clinical course and increased rate of resistance to immunosuppressive therapy $(19,20)$. Our patient had a very early onset at the age of 5 years as opposed to the average age of onset reported in the literature, between 10 and 12 years. Severe acute exacerbation of UC is a medical emergency. It is well known that the mortality rate is reduced considerably under pulsed corticosteroids (21). According to the European Crohn's and Colitis Organization the diagnosis of UC exacerbation in adults involves the presence of more than six bloody daily stools and one of the following criteria: tachycardia ( $\geq 90 \mathrm{bpm}$ ), temperature: above $37.8^{\circ} \mathrm{C}$, anemia ( $\mathrm{Hg}$ below $10.5 \mathrm{~g} / \mathrm{dl}$ ) or ESR above $30 \mathrm{~mm} / \mathrm{h}$ (22). In pediatric patients, the Pediatric Ulcerative Colitis Activity Index (PUCAI) is used to assess the severity of UC (23). This algorithm incorporates parameters such as abdominal pain, presence or absence of rectal bleeding, consistency and frequency of stools, respectively the patient's level of activity. Thus, a score above 65 is equivalent to a severe form. Furthermore, the lack of response to intravenous corticosteroids on day 5 of administration is the sign of steroid-refractory UC (23). Our patient benefited from pulsed therapy by accumulating a score of 80 .

Despite being part of the intestinal flora of infants, during childhood there is an progressive decrease in colonization with $\mathrm{CD}$. The age of 5 years is considered the threshold for maturation of the microbiome, after which the rate of CD carriers decreases (24). Taking into account the disturbances of the intestinal microbiome that are associated with IBD, we may consider that these patients have an increased risk for $\mathrm{CD}$. According to one study, the rate of asymptomatic $\mathrm{CD}$ carrier status in patients with IBD was higher in pediatric ages as compared to adults, i.e. $17 \%$ compared to $8.2 \%$ (25). In the case of our patient, we cannot say with certainty that he was not an asymptomatic carrier and that the pathogenicity of the CD was expressed in the context of exacerbation. Thus, it is difficult to say whether the $\mathrm{CD}$ was the trigger for exacerbation of UC or vice versa. Patients with both $\mathrm{CD}$ positive toxins may have more severe clinical symptoms (26), which has also been observed in the reported case. A study conducted in Beijing on 30 pediatric patients with IBD, showed that the incidence of both positive toxins is low, only one patient had both positive toxins (26).

$\mathrm{CD}$ infection has a higher rate of recurrence and morbidity in patients with IBD as compared to the general population, requiring prolonged hospitalization and escalation of therapy (27). However, patients with UC or Crohn's disease have a higher susceptibility to community acquired infection, unlike other 
patients who develop nosocomial CD infections (28). Periods of exacerbation of UC are frequently associated with this infection, making it difficult to differentiate the two entities since the symptoms are similar $(7,8,12)$. Most of the above symptoms were also present in our patient, the exacerbation occurring about 7 months after the diagnosis of IBD. In a recent pediatric study, $\mathrm{Hg}$, platelets, ESR and albumin had normal values at the time of diagnosis in $54 \%$ of mild UC cases, a situation not found in case of exacerbation (29). Similarly, our patient presented major changes in laboratory parameters in the context of exacerbation, with a slowly favorable evolution under treatment, but which can also be explained by $\mathrm{CD}$ infection. Laboratory parameters in $\mathrm{CD}$ infection are nonspecific consisting of leukocytosis (20-50,000/3) with important neutrophilia (30). Etiological diagnosis is based on highlighting the presence of CD toxins in feces (15). Similarly, our patient presented leukocytosis with neutrophilia, and the diagnosis was established by highlighting both toxins in the stools.

In order to establish optimal therapeutic conduct and assess the predictive capacity as well as facilitate the assessment of the disease, it is noted the usefulness of using the ATLAS score for CD colitis, including parameters such as age, temperature, number of leukocytes, albumin and the use of a systemic antibiotic (31). A study that assessed the severity of CD infection using this score showed that patients with a

\section{REFERENCES}

1. Birimberg-Schwartz L, Zucker DM, Akriv A, et al. Pediatric IBD Porto group of ESPGHAN. Development and Validation of Diagnostic Criteria for IBD Subtypes Including IBD-unclassified in Children: a Multicentre Study From the Pediatric IBD Porto Group of ESPGHAN. J Crohns Colitis. 2017;11(9):1078-84.

2. Benchimol El, Fortinsky KJ, Gozdyra P et al. Epidemiology of pediatric inflammatory bowel disease: A systematic review of international trends. Inflammatory Bowel Diseases 2011; 17(1):423-439.

3. Abraham BP, Kahn SA, Transition of Care in Inflammatory Bowel Disease. Gastroenterol Hepatol (NY). 2014;10(10):633-640.

4. Levine A, Griffiths A, Markowitz J et al. Pediatric Modification of the Montreal Classification for Inflammatory Bowel Disease: The Paris Classification. Inflammatory Bowel Disease 2011;17(6):1314-1321.

5. Molodecky NA, Soons IS, Rabi DM et al. Increasing incidence and prevalence of the inflammatory bowel diseases with time, based on systematic review. Gastroenterology 2012;142(1):46-54.

6. Siew C Ng, Hai Yun Shi, Hamidi N, et al. Worldwide incidence and prevalence of inflammatory bowel disease in the 21st century: a systematic review of population-based studies, The Lancet 2017; 390(10114):2769-2778

7. Kelsen J, Baldassano RN. Inflammatory bowel disease: The difference between children and adults. Inflammatory Bowel Diseases 2008;14(Suppl-2):S9-S11. score $\leq 3$ had an excellent prognosis, those with a score between 3 and 7 had a high probability of colectomy, and a score above 8 was associated with $100 \%$ mortality (32). According to this data, our patient accumulated a score of 2 . However, we found no studies to highlight the applicability of this score among pediatric patientsl. Metronidazole is considered the first treatment option for children with IBD and acute $\mathrm{CD}$ enterocolitis (33), without any studies to prove the need for another line of antibiotic therapy. In the case presented we preferred to administer it intravenously due to the patient's poor digestive tolerance, switching it orally after 7 days up to 14 days. Despite the fact that no resistance to Metronidazole was observed in pediatric CD strains (34), the evolution of our case, i.e. the framing in a severe form required the association of Vancomycin. Furthermore, the treatment instituted was a complex one that included antibiotics, pulsed therapy with SoluMedrol, administration of human Albumin, immunosuppressive treatment and symptomatic drugs.

\section{CONCLUSIONS}

The pediatric patient with UC and CDI remains a real challenge for pediatrician due to the alarming increase in morbidity, mortality and high healthcare costs. Parent's compliance in pediatric patients with IBD is essential regarding the prognosis of this pathology.

Conflict of interest: none declared Financial support: none declared

8. Jose FA, Garnett EA, Vittinghoff E et al. Development of Extraintestinal Manifestations in Pediatric Patients with Inflammatory Bowel Disease. Inflammatory Bowel Diseases 2009;15(1):63-68.

9. Turner D, Simon T, Griffiths AM et al. Consensus for Managing Acute Severe Ulcerative Colitis in Children: A Systematic Review and Joint Statement From ECCO, ESPGHAN, and the Porto IBD Working Group of ESPGHAN. American Journal of Gastroenterology 2011;106(4)574-588.

10. Adams DJ, Eberly ME, Rajnik M et al. Risk Factors for CommunityAssociated Clostridium difficile Infection in Children. The Journal of Pediatrics 2017;186:105-109.

11. Hourigan SK, MD, Sears CL, Hemker MO. Clostridium difficile Infection in Pediatric Inflammatory Bowel Disease. Inflamm Bowel Disease. 2016;22(4):1020-1025.

12. Kassper D, Fauci A, Harrison's Infectious Disease, 3rd Edition, New York: McGraw-Hill Education Medical, 2017;294-298.

13. Bradley SJ et al. 2020 Nelson's Pediatric Antimicrobial Therapy, 26th Edition. American Academy of Pediatrics, 2020;105-107.

14. Wultańska D, Banaszkiewicz A, Radzikowski A et al. Clostridium difficile infection in Polish pediatric outpatients with inflammatory bowel disease. European Journal of Clinical Microbiology \& Infectious Diseases 2010;29:1265-1270.

15. Heyman MB, Kirschner BS, Gold B et al. Children with early-onset inflammatory bowel disease (IBD): Analysis of a pediatric IBD consortium registry. The Journal of Pediatrics 2005;146(1):35-40. 
16. Muise AM, Snapper SB, Kugathasan S et al. The age of gene is discovery in very early onset inflammatory bowel disease. Gastroenterology-AGA 2012;143(2):285-288.

17. Ouahed J, Spencer E, Kotlarz D et al. Very Early Onset Inflammatory Bowel Disease: A Clinical Approach With a Focus on the Role of Genetics and Underlying Immune Deficiencies. Inflammatory Bowel Diseases 2020;26(6):820-842.

18. Kelsen JR, Sullivan KE, Shervin R et al. North American Society for Pediatric Gastroenterology, Hepatology, and Nutrition Position Paper on the Evaluation and Management for Patients With Very Early-onset Inflammatory Bowel Disease. J Pediatr Gastroenterol Nutr. 2020;70(3):389-403.

19. Seo JK. Pediatric inflammatory bowel disease (IBD): phenotypic, genetic and therapeutic differences between early-onset and adult-onset IBD. Korean J Ped Gastroenterol Nutr. 2011;14(1):1-25.

20. Cannioto Z, Berti I, Martelossi S et al. IBD and IBD mimicking enterocolitis in children younger than 2 years of age. European Journal of Pediatrics 2009;168:149-155.

21. Truelove SC, Witts LJ. Cortisone in ulcerative colitis; final report on a therapeutic trial. British Medical Journal 1955;2:1041-1048.

22. Travis SPL, Stange EF, Lémann $M$ et al. European evidence-based consensus on the management of ulcerative colitis: current management. Journal of Crohn's and Colitis 2008;2(1):24-62.

23. Turner D, Otley AR, Mack $D$ et al. Development, validation, and evaluation of a pediatric ulcerative colitis activity index: a prospective multicenter study. Gastroenterology 2007;133:423-432.

24. Kellermayer R. Burdening Questions About Clostridium difficile in Pediatric Inflammatory Bowel Diseases. Journal of Pediatric Gastroenterology and Nutrition 2015;60(4):421-422.

25. Mezoff E, Mann EA, Hart WK et al. Clostridium difficile Infection and Treatment in the Pediatric Inflammatory Bowel Disease Population. J Pediatr Gastroenterol Nutr. 2011;52(4):437-441.
26. Li D, Guo S, Guan DX, Zhao CN, Xu XW. [Infection rate and clinical characteristics of toxigenic Clostridium difficile in children with inflammatory bowel disease]. Zhonghua Er Ke Za Zhi. 2020 Jul 2;58(7):564-569.

27. Ananthakrishnan AN, McGinley EL, Binion DG. Excess hospitalisation burden associated with Clostridium difficile in patients with inflammatory bowel disease. Gut. 2008;57:205-210.

28. Issa M, Ananthakrishnan AN, Binion DG. Clostridium difficile and inflammatory bowel disease. Inflammatory Bowel Diseases 2008; 14(10):1432-1442.

29. Mack DR, Langton CR, Markowitz J et al. Laboratory Values for Children With Newly Diagnosed Inflammatory Bowel Disease. Pediatrics 2007;119(6):1113-9.

30. Cupșa A et al. Boli Infecțioase transmisibile. Editura Medicală Universitară, Craiova, 2006;10.53-10.55.

31. Cophra T, Miller M, Severson R et al. ATLAS-A Bedside Scoring System Predicting Mortality Due to Clostridium difficile Infection (CDI) in Elderly Hospitalized Patients (abs). Infectious Disease Society of America, 2010.

32. Hernández-García R, Garza-González E, Miller M et al. Application of the ATLAS score for evaluating the severity of Clostridium difficile infection in teaching hospitals in Mexico. The Brazilian Journal of Infectious Diseases 2015;19(4):399-402.

33. Gawronska A, Banasiuk M, Lachowicz D et al, Metronidazole or Rifaximin for Treatment of Clostridium difficile in Pediatric Patients with Inflammatory Bowel Disease: A Randomized Clinical Trial. Inflamatory Bowel Disease 2017;23(12):2209-2214.

34. Wultańska D, Woszczatyński PO, Pituch $\mathrm{H}$ et al. Survey of susceptibility of clinical Clostridium difficile strains isolated from patients hospitalised in different departments of paediatric hospital to antimicrobial agents. Med Dosw Mikrobiol. 2007;59(2):161-8. 